\section{Gategory}

Polymer-Supported Synthesis

\section{Key words}

polyhedral oligomeric silsesquioxane (POSS)

\section{Pd-diimine}

complexes

polymerization

SYNFACDinth
of the mont

Y. ZHANG, Z. YE* (LAURENTIAN UNIVERSITY, CANADA)

Homogeneous Polyhedral Oligomeric Silsesquioxane (POSS)-Supported Pd-Diimine Complex and Synthesis of Polyethylenes End-Tethered with a POSS Nanoparticle via Ethylene "Living" Polymerization

Chem. Commun. 2008, 1178-1180.

\title{
Preparation of Polyethylenes End-Tethered with POSS
}

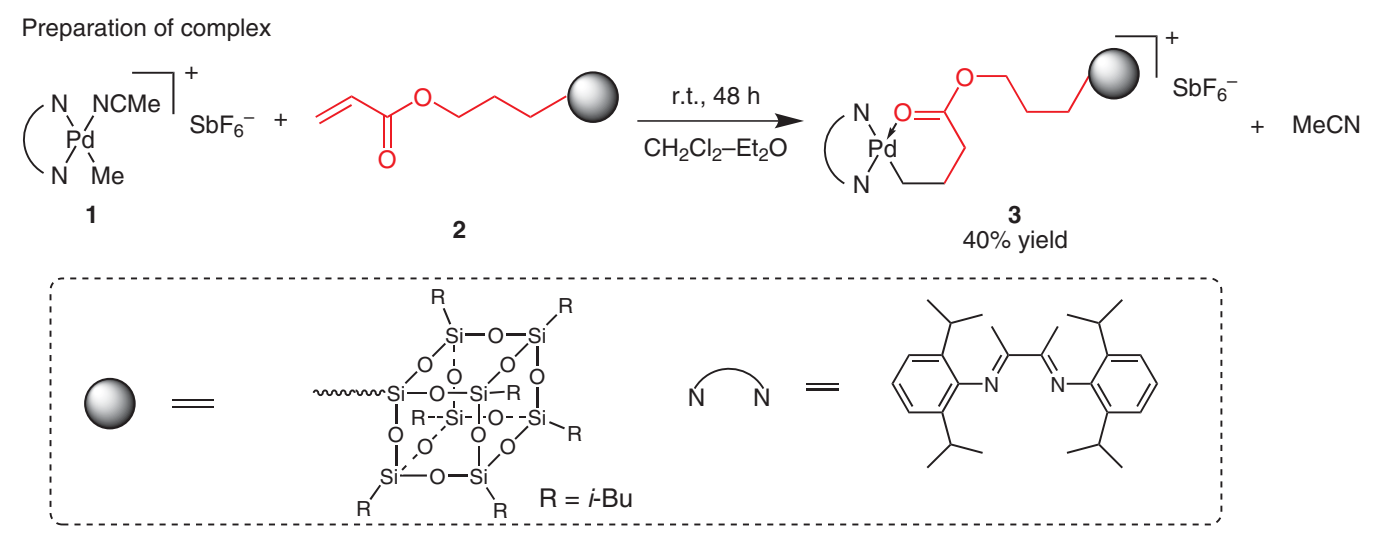

Ethylene polymerization
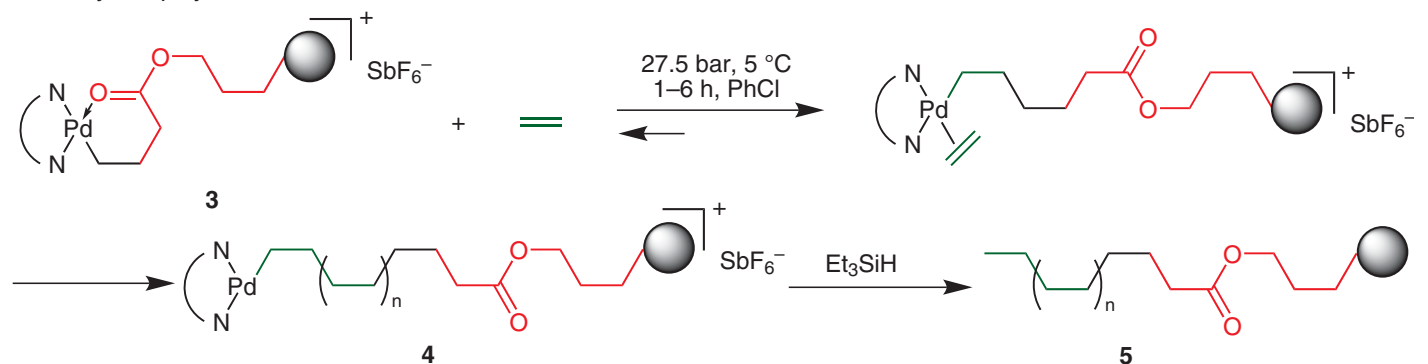

Monitoring results of $\mathbf{5}$

\begin{tabular}{|c|c|c|c|c|c|c|c|c|c|}
\hline $\begin{array}{l}\text { Time } \\
\text { (h) }\end{array}$ & $\begin{array}{c}\mathrm{Mn}, \mathrm{GPC} \\
(\mathrm{kg} / \mathrm{mol})\end{array}$ & PDI,GPC & $\begin{array}{l}\mathrm{Mn}, \mathrm{NMR} \\
(\mathrm{kg} / \mathrm{mol})\end{array}$ & $\begin{array}{l}\text { Branches } \\
\text { (per 1000C) }\end{array}$ & $\begin{array}{l}\text { Time } \\
\text { (h) }\end{array}$ & $\begin{array}{c}\mathrm{Mn}, \mathrm{GPC} \\
(\mathrm{kg} / \mathrm{mol})\end{array}$ & PDI,GPC & $\begin{array}{l}\mathrm{Mn}, \mathrm{NMR} \\
(\mathrm{kg} / \mathrm{mol})\end{array}$ & $\begin{array}{l}\text { Branches } \\
\text { (per 1000C) }\end{array}$ \\
\hline 1 & 15.2 & 1.11 & 11.2 & 89 & 4 & 43.5 & 1.12 & 36.3 & 87 \\
\hline 2 & 24.4 & 1.17 & 19.1 & 88 & 5 & 52.4 & 1.19 & 45.9 & 88 \\
\hline 3 & 36.6 & 1.14 & 29.6 & 88 & 6 & 56.4 & 1.17 & 51.6 & 87 \\
\hline
\end{tabular}

Significance: Preparation of a homogeneous polyhedral silsesquioxane (POSS)-supported Pddiimine complex $\mathbf{3}$ and telechelic polyethylenes end-tethered with POSS nanoparticles 5 was reported. Thus, complex $\mathbf{3}$ was prepared by the reaction of a Pd-diimine catalyst $\mathbf{1}$ with acryloisobutyl-POSS 2 at room temperature for 48 h (40\% yield). Ethylene polymerization was carried out with $\mathbf{3}$ at $5{ }^{\circ} \mathrm{C}$ under 27.5 bar in chlorobenzene followed by quenching $\mathbf{4}$ with triethylsilane to afford compound $\mathbf{5}$.
Comment: Monitoring results of $\mathbf{5}$ led to the following conclusions: The number average moleculer weight (Mn) was increased in proportion to time ( $1 \mathrm{~h} ; 15.2 \mathrm{~kg} / \mathrm{mol}$ vs $6 \mathrm{~h} ; 56.4 \mathrm{~kg} / \mathrm{mol}$ ). The polydispersity index (PDI) value was within 1.11-1.19. ${ }^{1} \mathrm{H}$ NMR measurements exhibited that these polymers were branched with ca. 88 branches per 1000 carbons. 